\title{
Spinal dural arteriovenous fistula: A rare cause of progressive myelopathy and bladder and bowel dysfunction
}

\author{
Hamza Sucuoğlü ${ }^{1,2}$ (D) Adem Aktürk ${ }^{3}$ (D) \\ 1'stanbul Gelișim University, School of Health Sciences, Istanbul, Turkey \\ ${ }^{2}$ Department of Physical Medicine and Rehabilitation, Private Nisa Hospital, Istanbul, Turkey \\ ${ }^{3}$ Department of Radiology, Private Nisa Hospital, Istanbul, Turkey
}

Received: October 01, 2018 Accepted: February 11, 2019 Published online: May 18, 2020

\begin{abstract}
Spinal dural arteriovenous fistula (SDAVF) is a rarely seen vascular lesion in the spinal cord and is often overlooked. If left untreated in the early stages, it is associated with severe morbidity and may lead to progressive myelopathy and bladder and bowel dysfunction. A 55-year-old male patient was admitted with complaints of lower extremity weakness, gait disorder, urinary retention, and stool retention. Based on physical examination and magnetic resonance imaging findings, a preliminary diagnosis of SDAVF was made. The diagnosis was confirmed by spinal angiography showing SDAVF on the left T6. Microsurgery was planned, once endovascular embolization failed. Although symptoms of progressive myelopathy and bladder and bowel dysfunction are rarely seen, SDAVF diagnosis should not be overlooked, and it should be kept in mind that early diagnosis and treatment prevent severe morbidities.
\end{abstract}

Keywords: Angiography, fistula, magnetic resonance imaging, myelopathy, rehabilitation, spinal cord.

Spinal dural arteriovenous fistula (SDAVF) is a vascular lesion caused by the interactions between the arterial and venous vascular systems of the spinal cord. ${ }^{[1,2]}$ It may induce abnormal flow of the blood from the arterial system to the venous system, venous hypertension, venous occlusion, intramedullary edema, and progressive myelopathy. ${ }^{[3-5]}$ The incidence of SDAVF is 5 to 10 in a million, and the majority of patients are male patients between their 60s and 70s. ${ }^{[4,5]}$

Since SDAVF is a rare entity with an unknown etiology, its diagnosis can be easily overlooked. ${ }^{[1,2]}$ To prevent permanent neurological disorders such as gait disorder, urinary retention, or stool retention, early diagnosis and treatment of SDAVF are critical. ${ }^{[2]}$ Although the diagnosis of SDAVF is possible with the help of the suspicion of the clinician and attention of the radiologist during magnetic resonance imaging (MRI), the gold standard for the diagnosis is selective spinal angiography. ${ }^{[2-5]}$ Angiography is used for localization of the vascular lesion and treatment. Microsurgery and endovascular embolization are effective methods for the treatment of SDAVF. ${ }^{[1,2]}$
In this article, we report a case of SDAVF presenting with progressive myelopathy and bladder and bowel dysfunction in the light of literature data.

\section{CASE REPORT}

A 55-year-old male patient was admitted with complaints of lower extremity weakness, gait and balance disorder, urinary retention, and stool retention for six months with an increasing severity. The patient was previously applied to a variety of hospitals and specialties for his symptoms; however, the definitive diagnosis was unable to be made. On physical examination, the muscle strength was $5 / 5$ in the upper extremities, $3 / 5$ in the left lower extremity and $4 / 5$ in the right lower extremity based on the Global Oxford Scale. There was no spasticity. The deep tendon reflexes of the bilateral patella and Achilles tendons were hyperactive. The Babinski reflex was not evoked in either feet. The Hoffman reflex was negative. Based on the sensory examination, left hypoesthesia was observed starting from the T8 level. The functional 

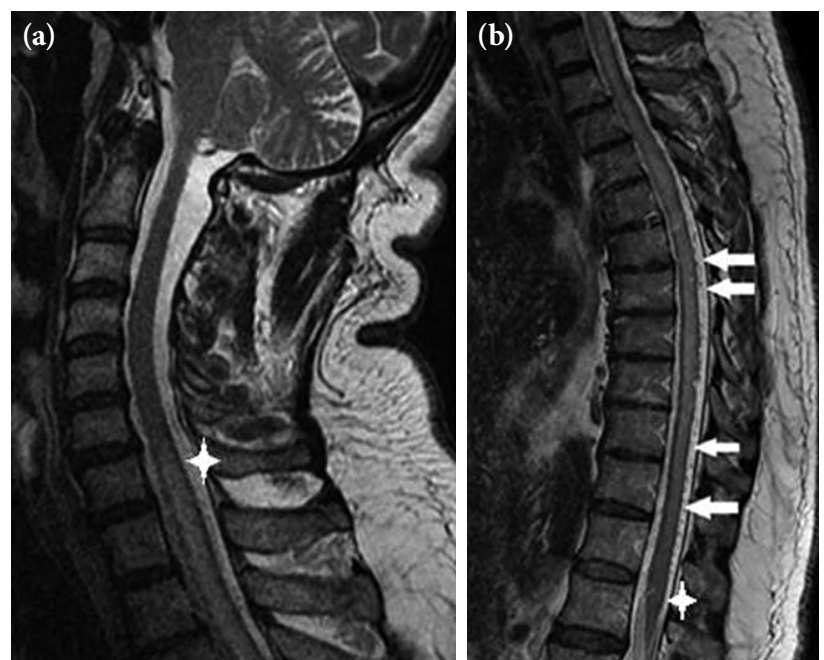

Figure 1. (a) T2-weighted cervical and (b) thoracic spine sagittal magnetic resonance imaging showing intramedullary increased signal from level of C6-7 disc (stars) to conus medullaris with associated expansion as well as signal void serpiginous structures (arrows) around spinal cord representing dilated vessels.

status of the patient was assessed using the modified Aminoff and Logue Scale of Disability (ALS), which grades gait, urinary incontinence, and fecal continence/ constipation. ${ }^{[6]}$ The total score of the ALS was 7/11, indicating moderate disability. The modified ALS sub-scores were as follows: gait-requires one cane or some support for walking "3"; urination-occasional incontinence or retention " 2 "; defecation-occasional incontinence or persistent constipation " 2 ". The results of the examinations of the patient which were carried out at external centers and biochemistry, complete blood count, sedimentation, and CRP values were all normal. The lower extremity needle and conduction electromyography and cranial MRI findings were also normal. Based on these findings, a whole spinal column MRI was performed, and the MRI of the structures lying in the central part from the C6-7 disc level to the conus medullaris and filling almost all parts of the medulla spinalis showed hypointensity on T1A, accelerated hyperintense myelopathic signal increase on T2A, and numerous serpiginous dorsal epidural flow voids in the cerebrospinal fluid adjacent to the spinal cord (Figure 1). This situation was evaluated as a SDAVF and, based on the angiography performed for accurate diagnosis and treatment purposes, SDAVF between the radicular artery and the perimedullary veins was found on the T6 level on the left (Figure 2a).

A written informed consent was obtained from the patient. Endovascular embolization was planned by the interventional radiology department; however, it failed.
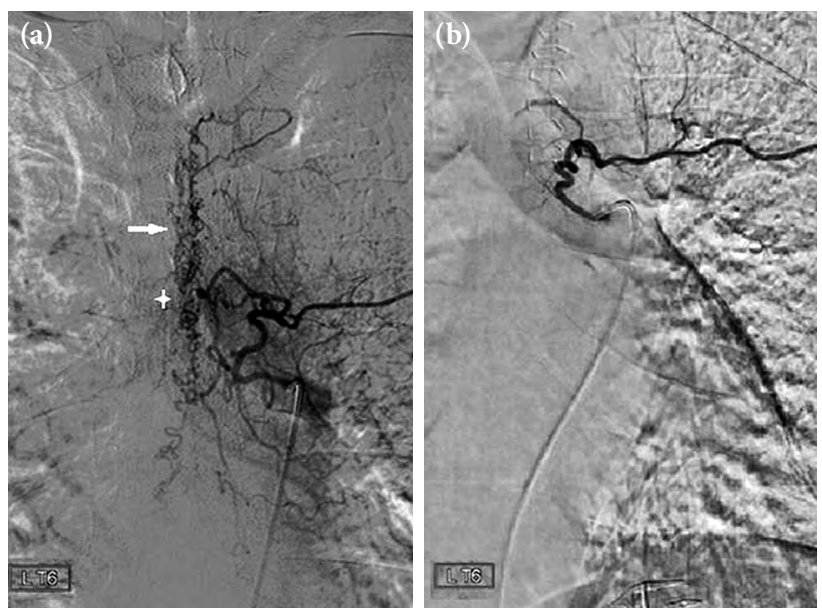

Figure 2. A spinal dural arteriovenous fistula on angiography (a) Before operation: During contrast injection at the left T6 level, abnormal filling of dilated epidural veins (arrow) and spinal dural arteriovenous fistula-dependent glomerular network-like image (star) between radial artery and perimedullary vein are seen. (b) After operation: Normal radial artery filling without abnormal venous enlargement following closure of fistula after surgical coagulation of perimedullary vein at the left T6 level.

Therefore, the patient was referred for microsurgery. During the surgical treatment, the fistula was closed and control angiography showed normal radial artery filling (Figure 2b). An outpatient rehabilitation program including passive and active assisted range of movement exercises, muscle strengthening exercises, neuromuscular electrical stimulation, and balance and coordination exercises was initiated from the first week after the operation. Additionally, the patient was consulted with the relevant clinics for bladder and bowel dysfunction.

\section{DISCUSSION}

Spinal dural arteriovenous fistula is a rarely seen spinal vascular malformation leading to severe morbidity, if left undiagnosed and untreated in the early stages. ${ }^{[2,7]}$ Blood reaches the spinal cord via spinal arteries (one at the anterior and two at the posterior) lying through the vertebral column, nerve roots, and radicular arteries before reaching the spinal cord. ${ }^{[2,8]}$ Anterior and posterior spinal veins exhibit a similar distribution to that of spinal arteries in the venous system and reach radicular veins, followed by the coronal venous plexus and the vena cava system. $^{[2,8]}$ Moreover, SDAVF occurs as a result of a direct connection between the radicular artery and the coronal venous plexus. Branches of the radicular artery merge with the venous system like a glomerular net at 
the fistula located in the dural envelope of the nerve root. ${ }^{[3]}$ As a result of the arterialization of the venous system by this way, venous hypertension, venous occlusion, ischemia, and edema develop, leading to progressive myelopathy. ${ }^{[3,4,7]}$ In our case presenting with edema and venous congestion, there was a distinctive myelopathy reaching from the $\mathrm{C} 6$ level to the conus medullaris and spinal cord extension.

Although SDAVF has no clear structural and hemodynamic classification, a series of classifications has been made to reflect its complex vascular anatomy. ${ }^{[9]}$ Currently, based on the most commonly used classification, spinal vascular malformations are divided into four main categories: Type I, dural arteriovenous fistula (DAVF); Type II, intramedullary glomus-type arteriovenous malformation (AVM); Type III, child or combined AVM, and Type IV, intradural perimedullary DAVF. ${ }^{[9,10]}$ About 60 to $80 \%$ of SDAVFs are located in the thoracic region, and most of these are unilaterally detected at the level of T7-12. ${ }^{[11,12]}$ This lesion is less frequently seen in the lumbar region and rarely seen at the cervical level. ${ }^{[7,12]}$ Bilateral or multiple SDAVF phenomena are extremely rare..$^{[1,12]}$ In our case, it was a unilateral, type I SDAVF at the left T6 level.

The majority of SDAVF patients are males over the age of $50 .^{[7,11]}$ The initial manifestations of this pathology are non-specific clinical symptoms, and median time to diagnosis ranges from 12 to 44 months. ${ }^{[13]}$ When myelopathy progresses, patients may present with complaints such as weakness in their lower extremity, gait disorder, sensory loss, and intestinal and urinary bladder dysfunction (incontinence or retention). ${ }^{[2,3,7,12]}$ The differential diagnosis of SDAVF includes polyneuropathies, demyelinating diseases, spinal neoplasms, and other spinal pathologies. ${ }^{[14]}$ Although gait disorder and bladder and bowel dysfunction symptoms were distinctive in our 55-year-old patient, the definitive diagnosis was unable to be made at external centers previously, and time from the onset of complaints to the diagnosis was six months. The reason for this delay may be the rareness of SDAVF and, the disease is frequently overlooked for this reason.

Although SDAVF can be detected by spinal MRI, which is the first step of examination for investigating progressive myelopathy, the gold standard for diagnosis is spinal angiography. ${ }^{[4,5]}$ The distinctive characteristics of SDAVF on MRI are as follows: based on progressive myelopathy, multiple observations of hyperintensity on the $\mathrm{T} 2$ sections lying through the vertebra segment to the conus medullaris, hypo-intense signals on the $\mathrm{T} 1$ sections, spinal cord extension based on edema, multiple intradural flow voids based on dilate intradural venous extensions and irregular cord surface images. ${ }^{[2,3,10]}$ Similar MRI findings were seen in our case. In addition, as in our case, the localization and level of the fistula and detailed characterization of the veins joining the fistula may be detected by spinal angiography. ${ }^{[1]}$

Although treatment is individually planned for each SDAVF patient, two effective treatment methods are microsurgery occlusion and endovascular embolization. ${ }^{[1,2,10]}$ The main goal of the treatment is to prevent abnormal blood flow by suspending the fistula itself or drainage vein from the proximal. Therefore, endovascular embolization is the first preferred method, as it is less invasive, allows starting rehabilitation earlier, and does not completely eliminate the option of having surgery. ${ }^{[2]}$ Although the success rates of endovascular and surgical treatments are similar, Hessler et al. ${ }^{[11]}$ reported that endovascular embolization was less successful compared to surgical treatment in 156 SDAVF patients for the previous 30 years. However, as a result of the recent developments in endovascular techniques, the success rate of embolization has reached about $77 \% .^{[12,15]}$

The prognostic factors of SDAVF vary in the literature. Young age, mild clinical course, and early diagnosis and treatment can be deemed as favorable prognostic factors. ${ }^{[2,16-18]}$ Contradictory results have been reported for the relationships between sex, fistula level, and short symptom time and prognosis. ${ }^{[12,18]}$

After the interventional treatment of SDAVF, early rehabilitation may positively contribute to the prognosis. ${ }^{[16]}$ According to the clinical condition of the patient, passive and active assisted range of movement exercises, muscle strengthening exercises (isometric or isotonic), progressive resistance exercises, neuromuscular electrical stimulation, balance and coordination exercises, lower limb robotic rehabilitation, and progressive ambulation training can be added to the rehabilitation program. ${ }^{[16]}$

In a retrospective study, Ofran et al. ${ }^{[18]}$ analyzed the rehabilitation results of eight SDAVF patients and reported that five patients were dependent on wheelchairs, despite a mean duration of two months of rehabilitation with a three-hour multidisciplinary approach including physiotherapy, occupational therapy, rehabilitation nursing, social workers, and psychological support. The importance of early diagnosis and early intervention rehabilitation 
indicated that success was related to functional ambulation potential. Additionally, most SDAVF patients reported that the rehabilitation process could be maintained in the outpatient setting; however, in patients with severe motor and functional disability, inpatient rehabilitation treatment could be required. In our case, outpatient rehabilitation started immediately after the intervention.

Clinical recovery continues during one-year follow-up after SDAVF treatment. ${ }^{[12,19]}$ Approximately $97 \%$ of patients show recovery ( 74 to $82 \%$ ) or stabilization (14 to $22 \%$ ) in terms of their motor findings. ${ }^{[12,19]}$ The recovery rate for sphincter dysfunctions is, however, lower and reported to be between 5 and $58 \% .^{[12,19,20]}$

In conclusion, SDAVF is an important spinal vascular pathology which should not be overlooked in the differential diagnosis of patients with progressive myelopathy, and bladder and bowel dysfunction. The etiopathogenesis of this disease has remained uncertain so far. Therefore, for a definitive diagnosis, it is required to recognize about MRI findings and conduct spinal angiography. Even though there are no precise guidelines for the treatment, endovascular embolization and surgery have been proven to be effective. Since it is a rare disease and easily overlooked, the suspicion of clinicians and attention of radiologists are of utmost importance to establish a definitive diagnosis. Early diagnosis and treatment are the most important prognostic factors for these patients in reducing morbidity.

\section{Acknowledgements}

We thank the, Private Nisa Hospital Physical Medicine and Rehabilitation Clinic, Radiology Clinic, for their contributions. We would also thank MD. Abdulkadir Asan, radiologist, who provided valuable contributions to the evaluation of the patient and to the collection of data in this study.

\section{Declaration of conflicting interests}

The authors declared no conflicts of interest with respect to the authorship and/or publication of this article.

\section{Funding}

The authors received no financial support for the research and/or authorship of this article.

\section{REFERENCES}

1. Iglesias Gordo J, Martínez García R. Spinalduralarteriovenous fistulas: the most frequent vascular malformations of the spinal cord. Radiologia 2018;60:237-49.

2. Geyik S, Gelebek F, Kaya T, Şirikçi A, Neyal M. Progresif paraparezi ve idrar retansiyonunun nadir bir sebebi: Spinal arteriovenöz fistül - Olgu sunumu. Türk Beyin Damar Hastalıkları Dergisi 2016;22:129-32.
3. Zakhary SM, Hoehmann CL, Cuoco JA, Hitscherich $\mathrm{K}$, Alam H, Torres G. A case report of spinal dural arteriovenous fistula: origins, determinants, and consequences of abnormal vascular malformations. Radiol Case Rep 2017;12:376-82.

4. Fugate JE, Lanzino G, Rabinstein AA. Clinical presentation and prognostic factors of spinal dural arteriovenous fistulas: an overview. Neurosurg Focus 2012;32:E17.

5. Koch C. Spinal dural arteriovenous fistula. Curr Opin Neurol 2006;19:69-75.

6. Aminoff MJ, Logue V. The prognosis of patients with spinal vascular malformations. Brain 1974;97:211-8.

7. Apostolova M, Nasser S, Kodsi S. A rare case of spinal dural arteriovenous fistula. Neurol Int 2012;4:e19.

8. Amato ACM, Stolf NAG. Anatomy of spinal blood supply. J Vasc Bras 2015;14:248-52.

9. Zozulya YP, Slin'ko EI, Al-Qashqish II. Spinal arteriovenous malformations: new classification and surgical treatment. Neurosurg Focus 2006;20:E7.

10. Jones BV, Ernst RJ, Tomsick TA, Tew J Jr. Spinal dural arteriovenous fistulas: recognizing the spectrum of magnetic resonance imaging findings. J Spinal Cord Med 1997;20:43-8.

11. Hessler C, Regelsberger J, Grzyska U, Illies T, Zeumer H, Westphal M. Therapeutic clues in spinal dural arteriovenous fistulas - a 30 year experience of 156 cases. Cent Eur Neurosurg 2010;71:8-12.

12. Ma Y, Chen S, Peng C, Wang C, Li G, He C, et al. Clinical outcomes and prognostic factors in patients with spinal dural arteriovenous fistulas : a prospective cohort study in two Chinese centres. BMJ Open 2018;8:e019800.

13. Jellema K, Tijssen CC, van Gijn J. Spinal dural arteriovenous fistulas: a congestive myelopathy that initially mimics a peripheral nerve disorder. Brain 2006;129:3150-64.

14. Jeng Y, Chen DY, Hsu HL, Huang YL, Chen CJ, Tseng YC. Spinal dural arteriovenous fistula: Imaging features and its mimics. Korean J Radiol 2015;16:1119-31.

15. Kirsch M, Berg-Dammer E, Musahl C, Bäzner H, Kühne D, Henkes H. Endovascular management of spinal dural arteriovenous fistulas in 78 patients. Neuroradiology 2013;55:337-43.

16. Güvendi E, Aşkın E, Aysin İ, Sarıkaya N, Koçyiğit H, Çakır $\mathrm{V}$, et al. Spinal dural arteriovenous fistula: A rare cause of progressive paraparesis. Turkish Journal of Geriatrics 2016;19:58-62.

17. Nagata S, Morioka T, Natori Y, Matsukado K, Sasaki T, Yamada T. Factors that affect the surgical outcomes of spinal dural arteriovenous fistulas. Surg Neurol 2006;65:563-8.

18. Ofran Y, Yovchev I, Hiller N, Cohen J, Rubin SA, Schwartz I, et al. Correlation between time to diagnosis and rehabilitation outcomes in patients with spinal dural arteriovenous fistula. J Spinal Cord Med 2013;36:200-6.

19. Saladino A, Atkinson JL, Rabinstein AA, Piepgras DG, Marsh WR, Krauss WE, et al. Surgical treatment of spinal dural arteriovenous fistulae: a consecutive series of 154 patients. Neurosurgery 2010;67:1350-7.

20. Behrens S, Thron A. Long-term follow-up and outcome in patients treated for spinal dural arteriovenous fistula. J Neurol 1999;246:181-5. 